\title{
Análise da influência do ultrassom de baixa intensidade na região de reparo ósseo em ratos sob ausência de carga
}

\author{
Analysis of the influence of low-intensity ultrasound the region of bone repair in rats under no load
}

Juliana de Carvalho Apolinário ${ }^{1}$, Willian Marinho Dourado Coelho² ${ }^{2}$ Mário Jefferson Quirino Louzada ${ }^{3}$

Estudo desenvolvido no Laboratório de Biofísica - Departamento de Apoio, Produção e Saúde Animal da Universidade Estadual Paulista (UNESP) Araçatuba (SP), Brasil.

1 Doutoranda do Programa Multicêntrico de Pós-Graduação em Ciências Fisiológicas da UNESP Araçatuba (SP), Brasil.

2 Doutorando do Programa de PósGraduação em Medicina Veterinária Preventiva da UNESP - Jaboticabal (SP), Brasil.

${ }^{3}$ Livre-docente; Docente do Departamento de Apoio, Produção e Saúde Animal na UNESP - Araçatuba (SP), Brasil.

ENDERECO PARA

CORRESPONDÊNCIA

Juliana de Carvalho Apolinário - Rua Clóvis Pestana, 793 - Dona Amélia CEP: 16050-680 - Araçatuba (SP), Brasil - E-mail: juapolinario@foa.unesp.br

\section{APRESENTACÃO}

jan. 2011

ACEITO PARA PUBLICAÇ̃̃O jul. 2011

FONTE DE FINANCIAMENTO FAPESP processo $n^{\circ}$ 07/54443-0

CONFLITO DE INTERESSES nada a declarar

APRESENTAÇÃO EM EVENTOS CIENTÍFICOS

$8^{\circ}$ Congreso Iberoamericano de Osteología y Metabolismo Mineral

Cumbre Iberoamericana de Osteoporosis

$3^{\circ}$ Congresso Brasileiro de Densitometria, Osteoporose e Osteometabolismo

1 ` Simpósio de Pós-Graduação em Ciência Animal

IX Semana de Divulgação Científica da UNESP - Araçatuba.

Parecer de aprovação do Comitê de Ética em Pesquisa da Faculdade de Odontologia da UNESP - campus Araçatuba $n^{\circ}$ 2007-002868.
RESUMO: Há evidências de que o ultrassom (US) de baixa intensidade pode acelerar a regeneração óssea. Este trabalho objetivou verificar a ação do US no defeito ósseo, criado experimentalmente em tíbias de ratos sob ausência de carga. Vinte Rattus novergicus albinus, Wistar adultos, divididos em: G1 $(n=10)$, grupo experimental de 15 dias sem suspensão, e G2 $(n=10)$, grupo experimental de 15 dias suspenso pela cauda, foram submetidos à osteotomia em ambas as tíbias e à aplicação do US, frequência de $1,5 \mathrm{MHz}$, ciclo de trabalho $1: 4,30 \mathrm{~mW} / \mathrm{cm}^{2}$, nas tíbias direitas por 12 sessões de 20 minutos. Após o sacrifício, as tíbias foram submetidas à análise da Densidade Mineral Óssea (DMO). Os resultados demonstraram DMO de $0,139 \pm 0,018 \mathrm{~g} / \mathrm{cm}^{2}$ para tíbia tratada; $0,131 \pm 0,009 \mathrm{~g} / \mathrm{cm}^{2}$ para tíbia controle no G1; e no G2 registrou-se $0,120 \pm 0,009 \mathrm{~g} / \mathrm{cm}^{2}$ para tíbia tratada e $0,106 \pm 0,017 \mathrm{~g} / \mathrm{cm}^{2}$ para tíbia controle. Houve diferença significante entre os grupos nos quais o G2 apresentou menor $\mathrm{DMO}$, o que demonstra que a suspensão prejudica a manutenção das propriedades ósseas, e entre as tíbias tratadas e controles do G2, demonstrando que o US acelerou o processo de reparo, concluindo que a impossibilidade do estímulo mecânico causada pela não deambulação em um processo de reparo ósseo pode ser minimizada pela ação do US. No G1, a aplicação do US não teve influência significante no aumento da DMO, talvez pelo fato dos animais já terem estímulo mecânico suficiente à formação óssea.

Descritores: densidade óssea; estimulação física; osteotomia; regeneração óssea.

ABSTRACT: There is evidence that the low-intensity ultrasound (US) can accelerate bone regeneration. This research studied the action of US in bone defect, created experimentally in the rat tibia under no load. Twenty Rattus norvegicus albinus, Wistar, divided into: $\mathrm{G} 1(\mathrm{n}=10)$, experimental group of 15 days without suspension, and G2 $(n=10)$ experimental group 15 days suspended by the tail, underwent osteotomy in both tibias and application of the US, frequency of $1,5 \mathrm{MHz}$, duty cycle 1:4, $30 \mathrm{~mW} /$ $\mathrm{cm}^{2}$, on the right tibia by 12 sessions of 20 minutes. After the sacrifice, tibias were subjected to analysis of Bone Mineral Density (BMD). The results demonstrated BMD of $0.139 \pm 0.018 \mathrm{~g} / \mathrm{cm}^{2}$ for treated tibia, $0.131 \pm 0.009 \mathrm{~g} / \mathrm{cm}^{2}$ for tibia control in the $\mathrm{G} 1$, and the $\mathrm{G} 2 \mathrm{had} 0.120 \pm 0.009 \mathrm{~g} / \mathrm{cm}^{2}$ for tibia and $0.106 \pm 0.017 \mathrm{~g} / \mathrm{cm}^{2}$ for control tibia. There were significant differences between groups where G2 had lower BMD, which shows that the suspension affect the maintenance of bone properties. There was significant difference between treated and control tibia of G2, showing that the US has accelerated the repair process, concluding that the failure of a mechanical stimulus caused by not walking in a process of bone repair can be minimized by the action of US. G1, in the application of US, did not have significant influence in increasing BMD, perhaps because the animals have already sufficient mechanical stimulus to bone formation.

Keywords: bone density; physical stimulation; osteotomy; bone regeneration. 


\section{INTRODUCÃO}

No Brasil, o contingente populacional com 65 anos ou mais de idade representava $6,53 \%$ da população total no ano de 2008. Estima-se que em 2025 o Brasil terá mais de 32 miIhões de pessoas consideradas idosas, o que chegará a 10,7\% da população, a tornando, desta forma, a sexta maior de idosos do mundo. Em 2050, esse índice ultrapassará os 22,71\% do total ${ }^{1}$. A partir dos 50 anos de idade, 30\% das mulheres e $13 \%$ dos homens podem sofrer algum tipo de fratura ${ }^{2}$ e a incidência deve quadruplicar nos próximos 50 anos em decorrência do aumento da expectativa de vida ${ }^{3}$.

Um complexo mecanismo de remodelação em nível celular, que incide de forma alternada entre formação e reabsorção óssea, conduz o tecido ósseo. Um ambiente desprovido de estímulos mecânicos produz efeitos diretos na estrutura óssea e altera sua função ${ }^{4,5}$. Assim, a conservação do metabolismo mineral ósseo normal depende de solicitações mecânicas geradas pelas contrações musculares e pela força da gravidade ${ }^{2}$.

Entre os fatores responsáveis pela manutenção do metabolismo mineral normal dos ossos está a compressão longitudinal exercida sobre eles, produzida pelo efeito da gravidade sobre o arcabouço. A pressão estimula o crescimento ósseo por aposição. A descarga de peso resulta em maior espessura do osso e maior densidade da diáfise ${ }^{6}$. Contudo, a exposição a ambientes de microgravidade e também a não deambulação, como a provocada pela presença de osteoporose em idosos ou por qualquer outra patologia, afetam diretamente o sistema músculo-esquelético, alterando seu metabolismo. Esta situação gera uma baixa tensão sobre os ossos e, por consequência, menor massa óssea será necessária para manter a integridade estrutural do esqueleto ${ }^{7}$.

As propriedades mecânicas dos ossos estão relacionadas ao seu conteúdo mineral e sua estrutura arquitetônica. Uma redução na massa óssea gera diminuição da resistência óssea, aumentando a suscetibilidade à fratura, que é determinada pelas propriedades biomecânicas do osso. A fratura, então, ocorre quando ao osso é imposta uma força maior do que ele poderia suportar ${ }^{8}$.

A proliferação celular e deposição de sais de cálcio determinam a regeneração do tecido ósseo lesado e restabelecem sua arquitetura e funcionalidade ${ }^{9}$ Q Quando a regeneração óssea não ocorre da forma esperada, torna-se necessário o implemento de métodos coadjuvantes, como o ultrassom (US) de baixa intensidade. Evidências em estudos in vitro e em animais sugerem que o US pulsado de baixa intensidade produz efeitos significativos osteoindutores, acelerando o processo de cura e a melhoria da força óssea ${ }^{10}$. O US, uma radiação acústica, é uma forma de energia mecânica que pode ser transmitida para o corpo como ondas de pressão de alta frequência. A energia acústica de US é produzida a partir de um cristal piezelétrico dentro de um transdutor, que emite ondas de alta frequência de pressão acústica transmitidas através dos tecidos do corpo por vibrações moleculares e colisões. As deformações micromecânicas produzidas por estas ondas de pressão nos tecidos do corpo podem resultar em eventos bioquímicos em nível celular e promover a formação de osso em um modo comparável com a resposta óssea ao estresse mecânico postulada pela lei de Wolff ${ }^{11,12}$.

A base fisiológica para a utilização do US como método terapêutico para o estímulo da osteogênese está nas propriedades biomecânicas do osso, pois tanto o impacto físico promovido pela descarga corporal quanto o promovido pelas ondas mecânicas do US provocam deformações nos ossos ${ }^{13,14}$.

Sabendo-se que em seres humanos a partir dos 50 anos de idade $30 \%$ das mulheres e $13 \%$ dos homens podem sofrer algum tipo de fratura, que a incidência de fraturas deve quadruplicar nos próximos 50 anos em decorrência do aumento da expectativa de vida e que grande parte destas fraturas impossibilitam a deambulação dos indivíduos, é objetivo deste estudo avaliar os efeitos do US no reparo ósseo por meio de análise densitométrica em tíbias de ratos submetidos à ausência de carga, tendo como hipótese que o US de baixa intensidade acelera a formação óssea.
METODOLOGIA

Grupos experimentais

Após aprovação do Comitê de Ética em Pesquisa da Faculdade de Odontologia da Universidade Estadual de São Paulo (UNESP) - Campus Araçatuba (Protocolo $\mathrm{n}^{\circ}$ 2007-002868), foram utilizados 20 ratos machos, Rattus novergicus albinus, linhagem Wistar, adultos com massa corporal média de $348 \pm 14 \mathrm{~g}$ fornecidos pelo Biotério da Faculdade de Odontologia da UNESP - Campus de Araçatuba e mantidos com ração comercial e água à vontade e sob temperatura controlada de $23^{\circ} \mathrm{C}$.

Os animais foram divididos em 2 grupos iguais: G1, submetidos à cirurgia para osteotomia que permaneceram livres em gaiolas individuais por 15 dias e foram tratados com US; e G2, submetidos à cirurgia para osteotomia e suspensos pela cauda por 15 dias e, concomitantemente, tratados com US. Os membros contra laterais (tíbia esquerda) foram os controles.

\section{Cirurgia}

Foram realizadas osteotomias cirúrgicas em ambas as tíbias, em região diafisária média, estando os animais sob anestesia geral com Ketamina (30 mg/kg) e Xilazina (3 $\mathrm{mg} / \mathrm{kg})$, via intramuscular.

Para a realização da osteotomia, a superfície anterior dos membros pélvicos dos animais, na região do terço médio, foi submetida à tricotomia. A seguir foi realizada incisão da pele no sentido longitudinal da tíbia e os músculos foram afastados com espátula, permitindo acesso ao osso. O defeito ósseo então foi obtido por perfuração com broca cirúrgica de $2 \mathrm{~mm}$ acoplada a motor de baixa rotação (uso odontológico), passando pelo periósteo e cortical óssea, até atingir o endósteo. Em todo o processo, soro fisiológico foi gotejado para a não necrose por aquecimento. Após a perfuração, o tecido muscular foi reposicionado e suturado e a pele também suturada ${ }^{15,16}$.

Após a cirurgia, por três dias os animais receberam Buprenorfina $(0,02 \mathrm{~mL}$ de 12 em 12 horas) via subcutânea, para analgesia pós-operatória, e Pentabiótico $(0,035 \mathrm{~mL})$ imediatamente após cirurgia como profilaxia antimicrobiana. 


\section{Técnica de suspensão}

A técnica de suspensão foi a mesma utilizada por Vicentini et al. ${ }^{17}$, divergindo no preparo da cauda do animal, que no presente estudo foi lavada com sabonete líquido antisséptico e teve a tintura de Benjoin substituída por lodopovedine tópico. A técnica de suspensão que, segundo Morey-Holton e Globus ${ }^{18}$, provoca um mínimo de estresse ao animal, foi realizada após a osteotomia com os animais (G2) ainda anestesiados.

\section{Tratamento com US}

Os animais foram imobilizados manualmente durante a fisioterapia e apenas o membro direito de todos dos grupos foi tratado com US pulsado de baixa intensidade, frequência de $1,5 \mathrm{MHz}$, ciclo de trabalho $1: 4,30 \mathrm{~mW} / \mathrm{cm}^{2}$, por 12 sessões de 20 minutos cada, servindo o membro esquerdo como controle. É a baixa intensidade $\left(30 \mathrm{~mW} / \mathrm{cm}^{2}\right)$ do US pulsado que promove aceleração à cicatrização óssea ${ }^{10}$.

O protocolo de tratamento se iniciou no primeiro dia após a osteotomia. As sessões de tratamento com US foram executadas de forma padronizada uma vez ao dia cumprindo-se um total de 12 sessões de tratamento, com 6 sessões consecutivas, pausa de 1 dia e mais 6 sessões consecutivas.

Após o período experimental, os animais foram anestesiados com Ketamina e Xilazina e então eutanasiados com Pentobarbital 1,5 mL (Tiopental ${ }^{\circledR}$ ) intravenoso e suas tíbias foram retiradas para análise densitométrica.

\section{Densitometria óssea}

Após a eutanásia, as tíbias foram desarticuladas, removidas, dissecadas e submetidas à análise densitométrica em densitômetro de dupla emissão de raios-X (DXA) - modelo DPX-Alpha, Lunar $^{\circledR}$ - com software especial para pequenos animais ${ }^{19}$. A região de reparo ósseo foi o local da análise.

\section{Análise estatística}

Os dados obtidos foram analisados estatisticamente pelo Teste $t$ de Student para comparar as tíbias tratadas e o controle em cada grupo e também entre os grupos, com o programa computacional

Tabela 1. Média e desvio padrão da densidade mineral óssea em g/ $\mathrm{cm}^{2}$ para tíbia controle e tratada do grupo 1 e do grupo 2

\begin{tabular}{lcc}
\hline & Tíbia controle & Tíbia tratada \\
\hline G1 & $0,131 \pm 0,009^{a}$ & $0,139 \pm 0,018^{\mathrm{b}}$ \\
G2 & $0,106 \pm 0,017^{\mathrm{a}, \mathrm{c}}$ & $0,120 \pm 0,009^{\mathrm{b}, \mathrm{c}}$ \\
\hline
\end{tabular}

Letras sobrescritas iguais implicam em diferença significante $(p<0,05)$

GraphPad InStat ${ }^{\circledR}$, versão 3.06. Os resultados foram apresentados como média \pm desvio padrão. O nível de significância utilizado foi de 5\% em todas as análises.

\section{RESULTADOS}

Os resultados estão demonstrados na Tabela 1 e são apresentados como média \pm desvio padrão para tíbia controle e tratada dos grupos 1 e 2 .

\section{DISCUSSÃO}

Houve diferença significante entre os grupos, com o G2 apresentando menor DMO em ambos os lados, o que demonstra que a suspensão prejudica a manutenção das propriedades ósseas.

O G1 não apresentou diferença na DMO entre a tíbia tratada e a controle, pois o mesmo não foi submetido à ausência de carga. Assim, a descarga mecânica necessária para estimular a piezoeletricidade já era suficiente para ativar o processo de remodelação óssea, diferente do G2, que estava sob suspensão, não tendo estímulo mecânico necessário para ativar a piezoeletricidade, sendo este estímulo mecânico substituído pelo estímulo do US, que em contato com o osso, provoca microdeformações, levando à remodelação óssea. A utilização do US como método terapêutico para o estímulo da osteogênese ocorre devido às propriedades biomecânicas do osso, pois o impacto físico promovido pelas ondas mecânicas do US provocam deformações nos ossos assim como o impacto promovido pela descarga corporal. A energia mecânica do US é transmitida pelos movimentos das partículas no meio pelo qual a onda se propaga. O meio irradiado oscila de acordo com a frequência do gerador ultrassônico, por efeito piezoelétrico, ao comprimir e expandir a matéria. As deformações micromecânicas ocorridas no tecido ósseo por ação do US são similares àquelas ocorridas sob carga mecânica e passam a agir como sinalizadores para formação do tecido ósseo ${ }^{13,14}$. Os resultados obtidos neste estudo para o grupo não suspenso (G1) concordam com Carvalho e Cliquet Jr ${ }^{20}$, que, analisando a ação do US pulsado de baixa intensidade durante 20 dias consecutivos por 20 minutos diários na região proximal dos fêmures de ratas osteopênicas, evidenciaram melhora da arquitetura dos ossos (análise qualitativa), mas não do conteúdo mineral (análise quantitativa), supondo que a análise quantitativa não tenha apontado diferenças devido ao fato de existir grande quantidade de tecido neoformado, o qual ainda não está totalmente mineralizado. Os autores comentam que a duração do tratamento com US de baixa intensidade talvez não tenha sido suficiente para possibilitar a total mineralização das regiões estimuladas, o que não gerou alterações de forma quantitativa, fato que pode ter influenciado os resultados do presente trabalho, uma vez que usamos somente análise quantitativa, além de termos utilizado menor tempo de tratamento quando comparados com outros estudos ${ }^{21-23}$.

Os achados desta pesquisa, na qual o G2 apresentou diferença significante entre as tíbias tratadas e controles, demonstra que o US acelerou o processo de reparo nestes ossos, fornecendo a informação de que a impossibilidade do estímulo mecânico causada pela não deambulação em um processo de reparo ósseo pode ser minimizada pela ação do US de baixa potência. A remodelação óssea, que ocorre também na tentativa de reparar fraturas, pode ser influenciada por forças mecânicas aplicadas ao esqueleto ${ }^{24}$.

Estas forças mecânicas e físicas são convertidas em sinais bioquímicos que se integram e geram respostas celulares $^{25}$, o que reforça a adaptação óssea a estímulos mecânicos. O tecido ósseo é referido como uma rede ampla na 
qual osteócitos atuam como células sensoriais, e osteoclastos e osteoblastos funcionam como células efetoras ${ }^{26}$. Os osteócitos são responsáveis por coordenar diferentes células ósseas em resposta à carga mecânica, por isso são relatados por estarem envolvidos na transdução do esforço de carga mecânica em resposta biológica ${ }^{27}$. Quando aplicadas cargas mecânicas sobre o tecido ósseo, há aumento do fluxo nos canalículos dos osteócitos, que sentem o fluxo do fluído e, consequentemente, produzem moléculas de sinalização óssea que regulam a reabsorção e formação óssea, mediadas pelos osteoclastos e osteoblastos, respectivamente ${ }^{28,29}$. É relatado também que no momento que a carga mecânica é imposta sobre o tecido ósseo, há liberação de Prostaglandinas, considerada um importante mediador bioquímico da carga mecânica sobre os ossos, e há envolvimento da sinalização do Óxído Nítrico ${ }^{30,31}$. A literatura também relata que forças mecânicas podem regular a expressão de Runx2, iniciando a diferenciação celular em células osteogênicas do periósteo, o que promove a regeneração óssea ${ }^{32}$.

Tais forças, quando necessário, podem ser supridas pela ação do US, como observado por Perry et al..$^{33}$ e neste estudo, que mostra que a formação óssea pode ser induzida por estímulo mecânico de baixa intensidade e o US pode ser a terapêutica útil para esta formação óssea.

\section{CONCLUSÕES}

O US acelerou o processo de reparo nos ossos dos animais submetidos à suspensão pela cauda, concluindo que a impossibilidade do estímulo mecânico causada pela não deambulação em um processo de reparo ósseo pode ser minimizada pela ação do US de baixa intensidade.

\section{REFERÊNCIAS}

1. IBGE, Instituto Brasileiro de Geografia e Estatística. Projeção da população do Brasil por sexo e idade - 1980-2050 - Revisão 2008. Estudos \& Pesquisas - Informação demográfica e socioeconômica 2008. 2010 jul. Disponível em http://www.ibge.gov.br/home/ estatistica/populacao/projecao_da_populacao/2008/ projecao.pdf.

2. NIH Consensus Development Panel on Osteoporosis Prevention, Diagnosis, and Therapy. Osteoporosis prevention, diagnosis, and therapy. 2001;285(6):785-95.

3. Rigss BL, Melton LJ. The worldwide problem of osteoporosis: insight afforded by epidemiology. Bone. 1995;17(5):505-11.

4. Holick MF. Perspective on the impact of weightlessness on calcium and bone metabolism. Bone. 1998;22(5):105-11.

5. Shimano MM. Microestruturas e propriedades mecânicas de ossos cortical e trabecular de ratos, após período de suspensão pela cauda e exercitação [tese]. Ribeirão Preto: Faculdade de Medicina de Ribeirão Preto, Universidade de São Paulo; 2006.

6. McArdle WD, Katch Fl, Katch VL. Fisiologia do exercício. Energia, nutrição e desempenho humano. Rio de Janeiro: Guanabara Koogan, 2003.

7. Johnson RB. The bearable lightness of being: bones, muscles, and spaceflight. Anat Rec. 1998;253(1):24-7.

8. Majumdar S, Kothari M, Augat P, Newitt DC, Lin JC, Lang $\mathrm{T}$, et al. High-resolution magnetic resonance imaging: three-dimensional trabecular bone architecture and biomechanical properties. Bone. 1998;22(5):445-54.

9. Doblaré M, García JM, Gómez MJ. Modelling bone tissue fracture and healing: a review. Eng Fract Mech. 2004;71(13-14):1809-40.
10. Martinez de Albornoz P, Khanna A, Longo UG, Forriol F, Maffulli N. The evidence of low-intensity pulsed ultrasound for in vitro, animal and human fractures healing. Br Med Bull. 2011.

11. Speed CA. Therapeutic ultrasound in soft tissue lesions. Rheumatology (Oxford). 2001;40(12):1331-6.

12. Baker KG, Robertson VJ, Duck FA. A review of therapeutic ultrasound: biophysical effects. Phys Ther. 2001;81(7):1351-8.

13. Naruse K, Mikuni-Takagaki Y, Azuma Y, Ito M, Oota T, Kameyama K, et al. Anabolic response of mouse bone-marrow-derived stromal cell clone ST2 cells to low-intensity pulsed ultrasound. Biochem Biophys Res Commun. 2000;268(1):216-20.

14. Kasturi G, Adler RA. Mechanical means to improve bone strength: ultrasound and vibration. Curr Rheumatol Rep. 2011;13(3):251-6.

15. Melo LGN. Avaliação do processo de reparo ósseo em cavidades cirúrgicas preenchidas com partículas de vidro bioativo associadas ou não à barreira de sulfato de cálcio: estudo histomorfométrico em tíbias de rato [dissertação]. Araçatuba: Faculdade de Odontologia, Universidade Estadual Paulista; 2003.

16. Riso NDM. Influência do laser terapêutico no reparo de defeito ósseo de ratos submetidos à ausência de carga: análise densitométrica [dissertação]. Araçatuba: Faculdade de Odontologia, Curso de Medicina Veterinária, Universidade Estadual Paulista; 2008.

17. Vicentini CR, Rosa RAC, Ciarlini LDRP, Santos PH, Louzada MJQ. Análise biomecânica em fêmures de ratos submetidos à ausência de carga e atividade física em esteira. Vet Zootec. 2007;14(1):62-71. 


\section{Referências (cont.)}

18. Morey-Holton ER, Globus RK. Hindlimb unloading of growing rats: a model for predicting skeletal changes during space flight. Bone. 1998;22(5):83-8.

19. Marques TM. Avaliação da densitometria óssea em fêmur de rato após consumo de álcool e fluoreto de sódio [dissertação]. Araçatuba: Faculdade de Odontologia, Universidade Estadual Paulista; 2008.

20. Carvalho DCL, Cliquet JR A. Low intensity ultrasound effects over osteopenic female rats bones. Acta Ortop Bras. 2003;11(1):17-24.

21. Azuma Y, Ito M, Harada Y, Takagi H, Ohta T, Jingushi S. Low-intensity pulsed ultrasound accelerates rat femoral fracture healing by acting on the various cellular reactions in the fracture callus. J Bone Miner Res. 2001;16(4):671-80.

22. Takikawa S, Matsui N, Kokubu T, Tsunoda M, Fujioka H, Mizuno K, Azuma Y. Low-intensity pulsed ultrasound initiates bone healing in rat nonunion fracture model. J Ultrasound Med. 2001;20(3):197-205.

23. Warden SJ, Fuchs RK, Kessler CK, Avin KG, Cardinal RE, Stewart RL. Ultrasound produced by a conventional therapeutic ultrasound unit accelerates fracture repair. Phys Ther. 2006;86(8):1118-27.

24.Szejnfeld VL. Osteoporose: diagnóstico e tratamento. São Paulo: Sarvier; 2000.

25. Huang H, Kamm RD, Lee RT. Cell mechanics and mechanotransduction: pathways, probes, and physiology. Am J Physiol Cell Physiol. 2004;287(1):C1-11.

26. Mi LY, Basu M, Fritton SP, Cowin SC. Analysis of avian bone response to mechanical loading. Part two: development of a computational connected cellular network to study bone intercellular communication. Biomech Model Mechanobiol. 2005;4(2-3):132-46.

27. Vezeridis PS, Semeins CM, Chen Q, Klein-Nulend J. Osteocytes subjected to pulsating fluid flow regulate osteoblast proliferation and differentiation. Biochem Biophys Res Commun. 2006;348(3):1082-8.

28. Santos A, Bakker AD, Klein-Nulend J. The role of osteocytes in bone mechanotransduction. Osteoporos Int. 2009;20(6):1027-31.

29. Cowin SC. The significance of bone microstructure in mechanotransduction. J. Biomech. 2007;40(1):S105-9.

30. Cheng B, Kato Y, Zhao S, Luo J, Sprague E, Bonewald $L F$, et al. $\operatorname{PGE}(2)$ is essential for gap junction-mediated intercellular communication between osteocytelike MLO-Y4 cells in response to mechanical strain. Endocrinology. 2001;142(8):3464-73.

31. Rubin J, Murphy TC, Zhu L, Roy E, Nanes MS, Fan X. Mechanical strain differentially regulates endothelial nitric-oxide synthase and receptor activator of nuclear kappa B ligand expression via ERK1/2 MAPK. J Biol Chem. 2003;278(36):34018-25.

32.Kanno T, Takahashi T, Ariyoshi W, Tsujisawa T, Haga $M$, and Nishihara T. Tensile mechanical strain up-regulates Runx2 and osteogenic factor expression in human periosteal cells: implications for distraction osteogenesis. J Oral Maxillofac. Surg. 2005;63(4):499-504.

33. Perry MJ, Parry LK, Burton VJ, Gheduzzi S, Beresford JN, Humphrey VF, et al. Ultrasound mimics the effect of mechanical loading on bone formation in vivo on rat ulnae. Med Eng Phys. 2009;31(1):42-7. 